International Mathematical Forum, 2, 2007, no. 45, 2231 - 2236

\title{
An Approximation to the Solution of Telegraph Equation by Adomian Decomposition Method
}

\author{
J. Biazar ${ }^{1}$ and H. Ebrahimi \\ Department of Mathematics, Faculty of Science \\ University of Guilan, P.O. Box 1914, Rasht, Iran
}

\begin{abstract}
Adomian decomposition method has been applied to solve many functional equations so far. In this article, this method is applied to solve a famous partial differential equation, called Telegraph equation.
\end{abstract}

Keywords: Adomian decomposition method; Telegraph equation; Adomian polynomials

\section{Introduction}

Consider an infinitesimal piece of telegraph wire as an electrical circuit, which consists of resistor of resistance $R d x$ and a coil of inductance $L d x$. If $i(x, t)$ is the current through the wire, the voltage across the resistor is $i R d x$ while that across the coil is $i_{t} L d x$. Denoting by $u(x, t)$ the voltage at position $x$ and time $t$. We have that the change in voltage between the ends of the piece of wire is

$$
d u=-i R d x-i_{t} L d x
$$

Suppose further that current can escape from the wire to ground, either through a resistor of conductance $G d x$ or through a capacitor of capacitance $C d x$. The amount that escapes through the resistor is $u G d x$. Because the charge on the capacitor is $q=u C d x$, the amount that escapes from the capacitor $u_{t} C d x$. In total

$$
d i=-u G d x-u_{t} C d x
$$

With dividing by $d x$ both sides of (1) and (2) and taking the limit $d x \longrightarrow 0$, we get the differential equations

$$
u_{t}+R i+L i_{t}=0
$$

\footnotetext{
${ }^{1}$ Corresponding author. e-mail: biazar@guilan.ac.ir
} 


$$
C u_{t}+G u+i_{x}=0
$$

By From $\frac{\partial}{\partial x}$ of (3) and $\frac{\partial}{\partial t}$ of (4), we have:

$$
\begin{aligned}
& u_{x x}+R i_{x}+L i_{t x}=0 \\
& C u_{t t}+G u_{t}+i_{x t}=0
\end{aligned}
$$

From (4) and (6), we obtain:

$$
\begin{gathered}
i_{x}=-C u_{t}-G u \\
i_{x t}=-C u_{t t}-G u_{t}
\end{gathered}
$$

Substituting (7) and (8) into (5) gives:

$$
u_{t t}+\left(\frac{G}{C}+\frac{R}{L}\right) u_{t}+\frac{G}{C} \frac{R}{L} u=\frac{1}{L C} u_{x x}
$$

Let $\alpha=\frac{G}{C}, \beta=\frac{R}{L}$ and $c^{2}=\frac{1}{L C}$. Thus

$$
u_{t t}+(\alpha+\beta) u_{t}+\alpha \beta u=c^{2} u_{x x}
$$

Where $u(x, t)$ is voltage at position $x$ and time $t$. Also by using $\frac{\partial}{\partial t}$ of $(3)$ and $\frac{\partial}{\partial x}$ of $(4)$, we have:

$$
i_{t t}+(\alpha+\beta) i_{t}+\alpha \beta i=c^{2} i_{x x}
$$

Where $i(x, t)$ is current through the wire. Equations (10) and (11) are called telegraph equation.

\section{Adomian decomposition method applied to telegraph equation}

Consider the following telegraph equation with the indicated initial conditions:

$$
\begin{gathered}
u_{t t}+(\alpha+\beta) u_{t}+\alpha \beta u=c^{2} u_{x x} \\
u(x, 0)=f(x) \\
\frac{\partial u(x, 0)}{\partial t}=g(x)
\end{gathered}
$$

Where $u$ can be voltage or current through the wire. For solving by Adomian decomposition method, pay attention to initial conditions consider operator $L_{t t}=\frac{\partial^{2}}{\partial t^{2}}$. The inverse operator of $L_{t t}$ is $L_{t t}^{-1}=\int_{0}^{t} \int_{0}^{t}() d t d$.$t and by applying$ the inverse operator to both sides of (12), we get:

$$
u(x, t)=u(x, 0)+\frac{\partial u(x, 0)}{\partial t} t+\int_{0}^{t} \int_{0}^{t}\left(c^{2} \frac{\partial^{2} u}{\partial x^{2}}-(\alpha+\beta) \frac{\partial u}{\partial t}-\alpha \beta u\right) d t d t
$$


Substituting (13) and (14) into (15), we have:

$$
u(x, t)=f(x)+g(x) t+\int_{0}^{t} \int_{0}^{t}\left(c^{2} \frac{\partial^{2} u}{\partial x^{2}}-(\alpha+\beta) \frac{\partial u}{\partial t}-\alpha \beta u\right) d t d t
$$

To solve equation (16) by Adomian decomposition method let, as usual in this method, the solution $u$ as the sum of a series

$$
u=\sum_{n=0}^{\infty} u_{n}
$$

So that the components $u_{n}$ will be determined recursive and the integrand on the right side as the sum of a series as:

$$
c^{2} \frac{\partial^{2} u}{\partial x^{2}}-(\alpha+\beta) \frac{\partial u}{\partial t}-\alpha \beta u=\Sigma_{n=0}^{\infty} A_{n}\left(u_{0}, u_{1}, \ldots, u_{n}\right)
$$

Where $A_{n}\left(u_{0}, u_{1}, \ldots, u_{n}\right)$ are called Adomian polynomials and should be computed. Adomian polynomials have been calculated by using an alternate algorithm for calculating Adomian polynomials [4]. Therefore we have:

$$
A_{n}\left(u_{0}, u_{1}, \ldots, u_{n}\right)=c^{2} \frac{\partial^{2} u_{n}}{\partial x^{2}}-(\alpha+\beta) \frac{\partial u_{n}}{\partial t}-\alpha \beta u_{n} \quad n=0,1,2, \ldots
$$

Substituting (17) and (19) into (16) yields to:

$$
\Sigma_{n=0}^{\infty} u_{n}=f(x)+g(x) t+\Sigma_{n=0}^{\infty} \int_{0}^{t} \int_{0}^{t}\left(c^{2} \frac{\partial^{2} u_{n}}{\partial x^{2}}-(\alpha+\beta) \frac{\partial u_{n}}{\partial t}-\alpha \beta u_{n}\right) d t d t
$$

To determine the components $u_{n}(n \geq 0)$ we first identify $u_{0}$ by terms that arise from the initial conditions at $t=0$. Second, the remaining components of $u(x, t)$ can be determined by procedure that each component is determined by using the preceding component.

In other words, the method introduces the following recursive relation:

$$
\begin{gathered}
u_{0}=f(x)+g(x) t \\
u_{n+1}=\int_{0}^{t} \int_{0}^{t}\left(c^{2} \frac{\partial^{2} u_{n}}{\partial x^{2}}-(\alpha+\beta) \frac{\partial u_{n}}{\partial t}-\alpha \beta u_{n}\right) d t d t \quad n=0,1,2, \ldots
\end{gathered}
$$

For the first few $n$, we have:

$$
\begin{gathered}
u_{1}=\left(\sum_{i=0}^{1} C_{i}^{1} c^{2 i}(-\alpha \beta)^{1-i} g^{(2 i)}(x)\right) \frac{t^{3}}{3 !}-(\alpha+\beta) g(x) \frac{t^{2}}{2 !}+\left(\sum_{i=0}^{1} C_{i}^{1} c^{2 i}(-\alpha \beta)^{1-i} f^{(2 i)}(x)\right) \frac{t^{2}}{2 !} \\
u_{2}=\left(\sum_{i=0}^{2} C_{i}^{2} c^{2 i}(-\alpha \beta)^{2-i} g^{(2 i)}(x)\right) \frac{t^{5}}{5 !}-2(\alpha+\beta)\left(\sum_{i=0}^{1} C_{i}^{1} c^{2 i}(-\alpha \beta)^{1-i} g^{(2 i)}(x)\right) \frac{t^{4}}{4 !}
\end{gathered}
$$




$$
\begin{gathered}
+(\alpha+\beta)^{2} g(x) \frac{t^{3}}{3 !}+\left(\sum_{i=0}^{2} C_{i}^{2} c^{2 i}(-\alpha \beta)^{2-i} f^{(2 i)}(x)\right) \frac{t^{4}}{4 !}-(\alpha+\beta)\left(\sum_{i=0}^{1} C_{i}^{1} c^{2 i}(-\alpha \beta)^{1-i} f^{(2 i)}(x)\right) \frac{t^{3}}{3 !} \\
u_{3}=\left(\sum_{i=0}^{3} C_{i}^{3} c^{2 i}(-\alpha \beta)^{3-i} g^{(2 i)}(x)\right) \frac{t^{7}}{7 !}-3(\alpha+\beta)\left(\sum_{i=0}^{2} C_{i}^{2} c^{2 i}(-\alpha \beta)^{2-i} g^{(2 i)}(x)\right) \frac{t^{6}}{6 !} \\
+3(\alpha+\beta)^{2}\left(\sum_{i=0}^{1} C_{i}^{1} c^{2 i}(-\alpha \beta)^{1-i} g^{(2 i)}(x)\right) \frac{t^{5}}{5 !}-(\alpha+\beta)^{3} g(x) \frac{t^{4}}{4 !} \\
+\left(\sum_{i=0}^{3} C_{i}^{3} c^{2 i}(-\alpha \beta)^{3-i} f^{(2 i)}(x)\right) \frac{t^{6}}{6 !}-2(\alpha+\beta)\left(\sum_{i=0}^{2} C_{i}^{2} c^{2 i}(-\alpha \beta)^{2-i} f^{(2 i)}(x)\right) \frac{t^{5}}{5 !} \\
+(\alpha+\beta)^{2}\left(\sum_{i=0}^{1} C_{i}^{1} c^{2 i}(-\alpha \beta)^{1-i} f^{(2 i)}(x)\right) \frac{t^{4}}{4 !}
\end{gathered}
$$

Therefore the general term will be as:

$$
\begin{aligned}
& u_{n}=\sum_{k=1}^{n+1}\left((-1)^{n-k+1} C_{k-1}^{n}(\alpha+\beta)^{n-k+1}\left(\sum_{i=0}^{k-1} C_{i}^{k-1} c^{2 i}(-\alpha \beta)^{k-i-1} g^{(2 i)}(x)\right) \frac{t^{n+k}}{(n+k) !}\right) \\
& +\sum_{k=1}^{n}\left((-1)^{n-k} C_{k-1}^{n-1}(\alpha+\beta)^{n-k}\left(\sum_{i=0}^{k} C_{i}^{k} c^{2 i}(-\alpha \beta)^{k-i} f^{(2 i)}(x)\right) \frac{t^{n+k}}{(n+k) !}\right) \quad n=0,1,2, \ldots
\end{aligned}
$$

And the solution is:

$$
\begin{aligned}
& u(x, t)=\sum_{n=0}^{\infty} \sum_{k=1}^{n+1}\left((-1)^{n-k+1} C_{k-1}^{n}(\alpha+\beta)^{n-k+1}\left(\sum_{i=0}^{k-1} C_{i}^{k-1} c^{2 i}(-\alpha \beta)^{k-i-1} g^{(2 i)}(x)\right) \frac{t^{n+k}}{(k+n) !}\right) \\
& +\sum_{n=0}^{\infty} \sum_{k=1}^{n}\left((-1)^{n-k} C_{k-1}^{n-1}(\alpha+\beta)^{n-k}\left(\sum_{i=0}^{k} C_{i}^{k} c^{2 i}(-\alpha \beta)^{k-i} f^{(2 i)}(x)\right) \frac{t^{n+k}}{(k+n) !}\right)
\end{aligned}
$$

The exact solution of equation (12) is now entirely determined. However, in practice all terms of the series $\Sigma_{n=0}^{\infty} u_{n}$ can not be determined; thus we take an approximation of the solution form the truncated series $\varphi_{n}=\sum_{i=0}^{n-1} u_{i}$ with $\lim _{n \rightarrow \infty} \varphi_{n}=u$.

\section{Numerical results}

example 1: Consider the telegraph equation with the initial conditions:

$$
u_{t t}+(\alpha+\beta) u_{t}+\alpha \beta u=c^{2} u_{x x}
$$




$$
\begin{aligned}
& u(x, 0)=\sin x \\
& \frac{\partial u(x, 0)}{\partial t}=0
\end{aligned}
$$

By using Adomian decomposition method we have:

$$
\begin{gathered}
u_{0}=\sin x \\
u_{n}=(-1)^{n} \sin x \sum_{k=1}^{n}\left(C_{k-1}^{n-1}(\alpha+\beta)^{n-k}\left(c^{2}+\alpha \beta\right)^{k} \frac{t^{n+k}}{(n+k) !}\right) \quad n=1,2,3, \ldots
\end{gathered}
$$

Thus, the solution is:

$$
u(x, t)=\sin x\left(\sum_{n=0}^{\infty} \sum_{k=1}^{n}\left((-1)^{n} C_{k-1}^{n-1}(\alpha+\beta)^{n-k}\left(c^{2}+\alpha \beta\right)^{k} \frac{t^{n+k}}{(n+k) !}\right)\right)
$$

For $\alpha=\beta=c=1$ seven-terms approximation to the solution will be as follows:

$$
\begin{gathered}
u(x, t) \approx \sin x\left(1-t^{2}+\frac{2}{3} t^{3}-\frac{1}{6} t^{4}+\frac{1}{90} t^{6}-\frac{1}{315} t^{7}\right. \\
\left.+\frac{1}{280} t^{8}+\frac{4}{2835} t^{9}+\frac{19}{113400} t^{10}+\frac{1}{124740} t^{11}+\frac{1}{7484400} t^{12}\right)
\end{gathered}
$$

example 2: Let

$$
\begin{gathered}
u(x, 0)=e^{x} \\
\frac{\partial u(x, 0)}{\partial t}=x \\
\alpha=2 \quad \beta=3 \quad c=4
\end{gathered}
$$

By using (20) seven-terms approximation to the solution would be as:

$$
\begin{gathered}
u(x, t) \approx \frac{3}{400400} x t^{13}+\left(\frac{3}{6160} x+\frac{625}{299376} e^{x}\right) t^{12}+\left(\frac{369}{30800} x-\frac{3125}{49896} e^{x}\right) t^{11} \\
+\left(\frac{47}{336} x+\frac{1625}{2268} e^{x}\right) t^{10}+\left(\frac{879}{1120} x-\frac{18125}{4536} e^{x}\right) t^{9}+\left(\frac{57}{32} x+\frac{375}{32} e^{x}\right) t^{8} \\
+\left(\frac{2059}{5040} x-\frac{1375}{72} e^{x}\right) t^{7}+\left(-\frac{133}{144} x+\frac{1475}{72} e^{x}\right) t^{6}+\left(\frac{211}{120} x-\frac{75}{4} e^{x}\right) t^{5} \\
+\left(-\frac{65}{24} x+\frac{175}{12} e^{x}\right) t^{4}+\left(\frac{19}{6} x-\frac{25}{3} e^{x}\right) t^{3}+\left(\frac{-5}{2} x+5 e^{x}\right) t^{2}+x t+e^{x}
\end{gathered}
$$

\section{Conclusion and Discussion}

The Adomian decomposition method is a powerful method, which has provided an efficient potential for the solution of physical applications modeled by nonlinear differential equations $[1,2,3]$. The main goal of article has been to derive an approximation to the solution of telegraph equation. We have achieved this goal by applying Adomian decomposition method. In Examples, the approximation can be obtained to any desired number of terms to increase the level of accuracy. Also For computations we used the package Maple 9. 


\section{Reference}

[1] Adomian, G., Nonlinear Stochastic Systems Theory and Applications to Physics, Kluwer Academic Press, (1989).

[2] Adomian, G., Solving Frontier Problems of Physics The Decomposition Method, Kluwer Academic Press, (1994).

[3] Adomian, G., Bellman, R., Partial Differential Equations, Reidel Publishing, (1985).

[4] Biazar, J., Babolian, E., Nouri, A., Islam, R., An Alternate Algorithm for Computing Adomian Polynomial in Special Cases, Applied Mathematics and Computing, 28(2-3),PP.523-529,(2003)

Received: June 1, 2006 